\title{
Real-time Vehicle Surveillance System Based on Image Processing and Short Message Service
}

\author{
Agustinus Deddy Arief Wibowo ${ }^{1}$, Rudi Heriansyah ${ }^{2}$ \\ ${ }^{1}$ Monster Technologies Malaysia Sdn Bhd, Kuala Lumpur, Malaysia \\ ${ }^{2}$ Computer Engineering Technology Section, Malaysian Institute of Information Technology, Universiti Kuala \\ Lumpur, Malaysia \\ ${ }^{1}$ deddyariefl@gmail.com, ${ }^{2}$ rudi@unikl.edu.my
}

\begin{abstract}
This paper proposes a real-time vehicle surveillance system based on image processing approach tailored with short message service. A background subtraction, color balancing, chain code based shape detection, and blob filtering are used to detect suspicious moving human around the parked vehicle. Once detected, the developed system will generate a warning notification to the owner by sending a short message to his mobile phone. The current frame of video image will also be stored and be sent to the owner e-mail for further checking and investigation. Last stored image will be displayed in a centralized monitoring website, where the status of the vehicle also can be monitored at the same time. When necessary, the stored images can be used during investigation process to assist the authority to take further legal actions.
\end{abstract}

Keywords: parked-vehicle, real-time surveillance, short message service, video image

\section{INTRODUCTION}

Feeling secure is an important part of every human life, and that is why it is essential for human to feel secure and safe. Part of those feeling secure is about being safe about their belongings. This paper will be dealing with one of those belongings, which is a vehicle. In order to do so, this paper will implement image processing to create such a real-time guarded vehicle.

The vehicle security level itself is not showing a really good numbers recent years. Fig. 1 shows the number of stolen vehicle in Malaysia for the last two years, which indicates the number of stolen vehicle considerably high [1]. The private cars are stolen more than the commercial cars. This situation urges a necessity to implement or add any new features into current vehicle security system in order to minimize this very critical unwanted situation.

Numerous methods have been proposed for an antitheft system for a vehicle. Anti-theft system based on vehicular sensor network is one of the approach [2]. In this system, any unauthorized vehicle movements are detected by car sensor that form sensor network. Once detected, an alert will be sent to the local base station which send warning to the security office. However, this system may not work well in a sparse parking area in a sense that sensors cannot generate a good enough network for protection purposes. Hori et al. [3] employs $24 \mathrm{GHz}$ frequency band intrusion sensor in their anti-theft system that installed inside the vehicle to sense object movement. However, this system is still in its development phase and not in a practical use yet.

In another system, a theft will be detected when unlocked the door of the car. Once the engine is started, a portable device holding by the owner will start to track the car using GPS. Thus, the car owner will be able to retrieve the car as soon as possible. The system uses a dedicated device to enable the system working properly [4].

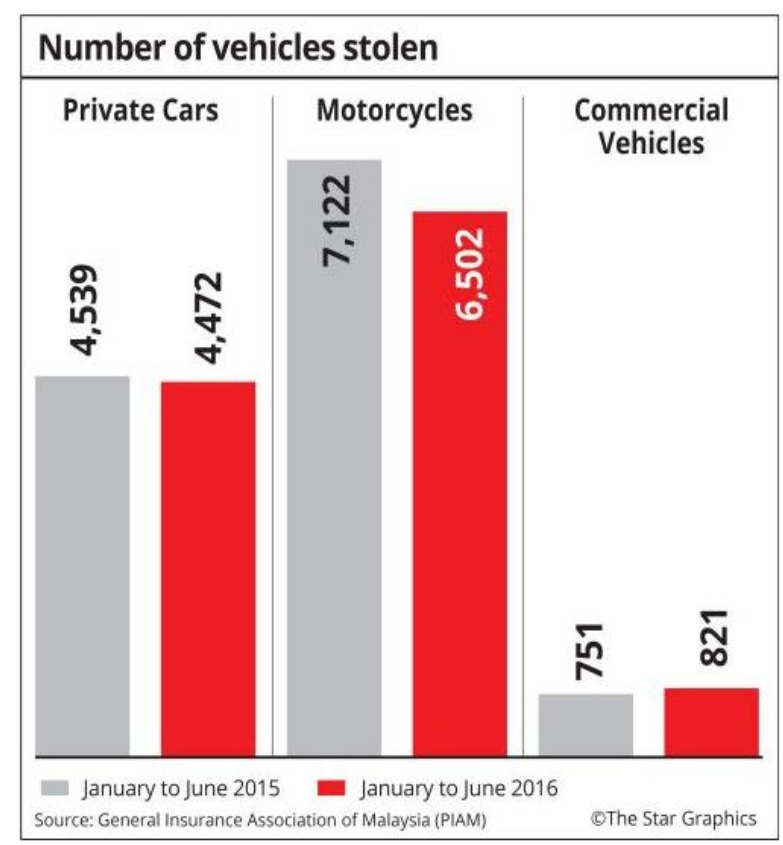

Fig. 1 Statistics of vehicle stolen cases in Malaysia for the last twenty years 
A face detection and finger print recognition are combined for car intrusion avoidance system [5]. Face of any driver will be captured by installed webcam at the sun shield upon closing of the car's door. The face image will be compared with the owner's face image in the database. In addition, fingerprint of the driver will be scanned although it is not mentioned clearly in how the process is carried out for this purpose. Engine can only ignite if both face and fingerprint match with those to the owner. The drawback of the system is that it can do nothing when the theft is supposed to steal stuffs inside the vehicle for example by breaking the windows.

This paper comes up with a bit different approach, the system developed will implement motion detection algorithm. From which, the system will extract several parameters to determine and take decision whether the situation in the inspected scenes requires further action or not. The system will record and capture moving human near outside the vehicle that may do some suspicious activities to the vehicle. An intrusion is considered taken place for example when someone is breaking the glass windows of the vehicle. Once the system identified that further action is required, then the system will notify the owner of the vehicle through short message service (SMS) and e-mail. Section 2 describes approaches for the system in which the implementation will be discussed in Section 3. Section 4 concludes and discusses further development of the proposed system.

\section{METHOD}

The proposed system consists of two main modules: (1) image processing module and (2) database module. In the first module, moving human will be detected by motion detection technique. Any detected movement of suspicious human will be recorded in the database and certain message will be sent to the owner mobile phone and captured image will be sent to the owner's e-mail.

\section{A. Image Processing Module}

Stages for this module is shown in Fig. 2. Input video are real-time image frames captured by webcam installed inside the vehicle. The input of this system is in form of video streams, which captured by a video acquisition device with video rate fifteen frames per second. The system employed two video acquisition device which will be used to capture scene from left and right side of the vehicle. The video is captured in RGB color space [6] and 480x640 resolutions. Video acquisition device will feed this stream of video to the first stage of the system, which is pre-processing stage.
This stage will processes stream of video that coming from video acquisition device. For simulation, the system will read a video that has been recorded before. The video will be processed frame by frame at this stage until final stage, where the system will determine whether it is necessary to invoke the warning mechanism. At this stage as well, the system will perform necessary action to prepare the data for processing. One of the preprocessing step is convert incoming frame which in RGB color space to grayscale. The image is converted into grayscale image for more efficient processing. Since RGB color space uses three color channels where grayscale only uses one channel, it will be more efficient to process the image in grayscale [7-8]. In order to illustrate the flow of pre-processing stage is shown in Fig. 3.

Region of interest (ROI) also need to be defined in this stage. ROI here defines area on the image that will be processed by the system. The usage of ROI in this system is to improve the efficiency of the system. By this ROI, the system will only processes pixels that located inside it. In this method as shown in Fig. 4, the ROI will be vehicle's window area, where the movement surrounding the car will be monitored by the system through webcam inside the vehicle. This ROI also can be used as reference line to determine whether the object is located near or beside the vehicle or located in a safe distance from the vehicle.

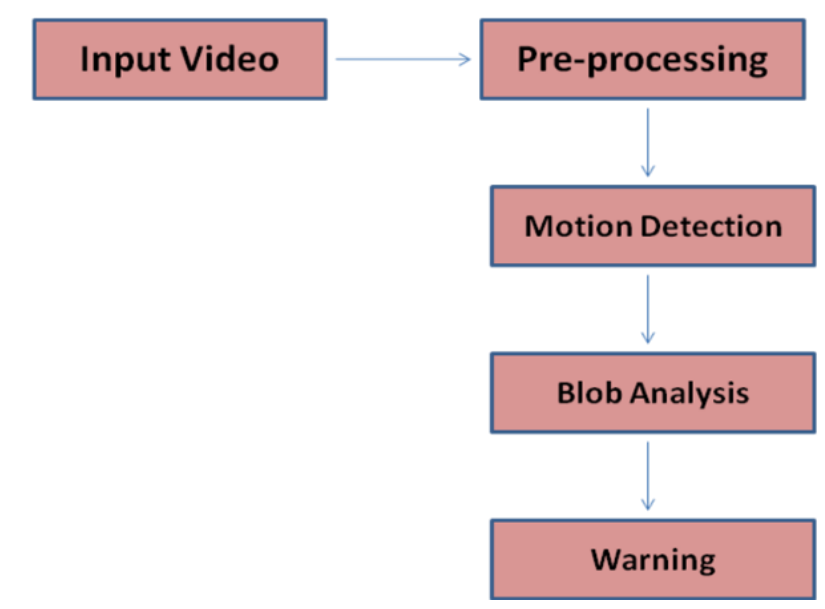

Fig. 2 Stages in image processing module

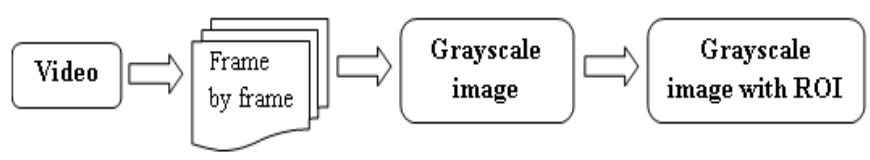

Fig. 3 Pre-processing flow 


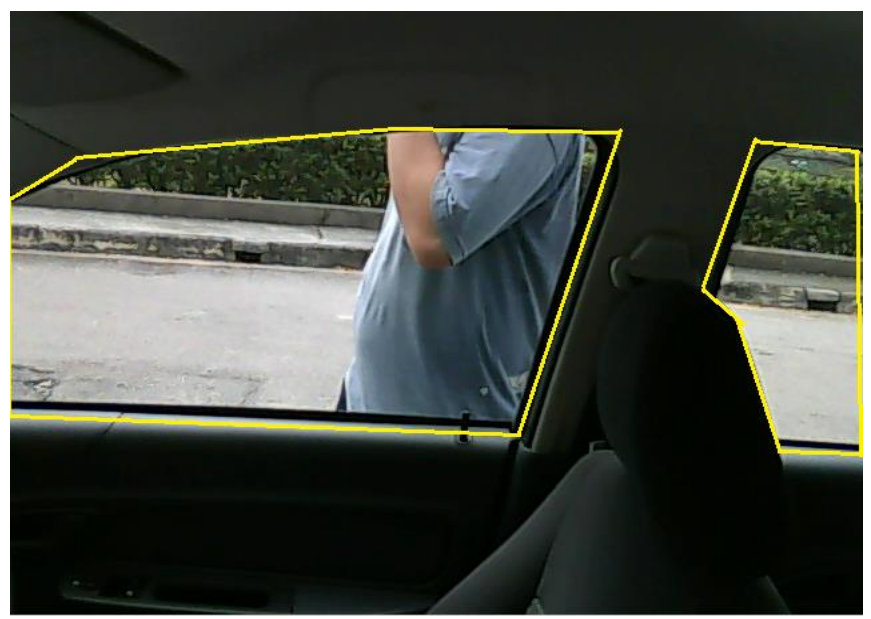

Fig. 4 Selection of ROI

After pre-processing, the system will detect if there is a movement in the incoming scene. This stage receives input from previous stage in terms of background image and current frame that already converted into grayscale. Before the background image and current frame converted into grayscale image, these image went through color balancing process [9]. Color balancing process is done to reduce or minimize the effect of different level of light intensity on the image [10]. Color balancing is important in motion detection stage as area with different light intensities can be detected as moving object on the scene. This stage also gets information about pre-defined region of interest from pre-processing stage. The system will use this information in order to determine if there is a movement in the current scene. Background subtraction is used for motion detection purpose [11].

As shown in the figure, color balancing is carried out before background subtraction taken place. This color balancing is using gray world assumption which stated that average of three color channels is neutral gray [12]. Background subtraction is sensitive to change in light intensity, therefore color balancing is essential step before background subtraction. The system will use the background image as reference and compare it with current frame by subtract them, creating a residual image which indicate the difference the current frame and the background image. Any residual images after subtraction indicates that there is a movement in the scene.
The residual image obtained in motion detection stage is fed into blob analysis stage. At this stage, the system will perform blob analysis on the residual image. Residual image will go through blob filtering, morphological operations [13], blob monitoring and draw boundaries around the blobs. Those steps are necessary for the system in order generate output that will be used in the warning generation stage.

Blob analysis steps are shown in Fig. 5. At the first step, the residual image will dilated and eroded respectively [14]. Erosion operation is done to remove any unwanted noise in the residual image. While dilation operation is aiming to fill the holes inside blobs, result in more solid blobs. Solid blob is useful as it will represent the body of the moving object in the scene which will marked by green line around its boundaries.

After the system performed morphological operations, blobs that formed will go through filtering process. This filtering process is done to further remove any noises that still remain in the scene after morphological operation. In this filtering process, the system will use the size of the blobs, ratio, and the position of the blobs. Blobs with smaller size than threshold value will be removed. It is done with assumption that blobs with small size is not part of the moving object. Blobs filtering also used ratio between height and width to filter the blobs. Blobs with the ratio in which the height and the width that below the threshold will consider as noise and then be removed.

The other criteria that used to filter the blobs is position of blobs. When a blob does not intersect with lower boundary of ROI, the respective blob will be filtered out. This filtering criteria is used to filter object that not located near to the vehicle which can be safely assume will has less potential of any criminal activity. Blob will also be filtered out if the blob intersects with upper boundary of ROI. Filtering process using blobs position is done by checking where the blob is located, whether it is on left part of the ROI or right part of ROI. Upper and lower boundary of respective ROI part will be used to check whether the blob intersects with upper or lower boundaries. The intersection checking also uses certain range of range to add flexibility to the system.

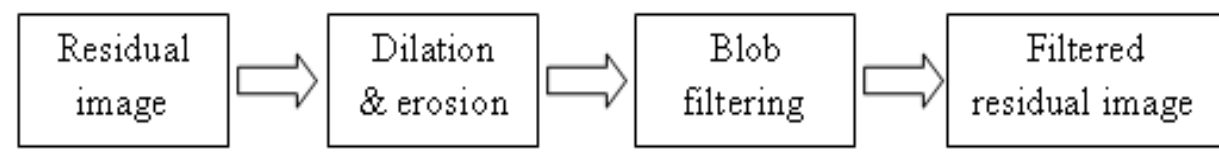

Fig. 5. Blob analysis 


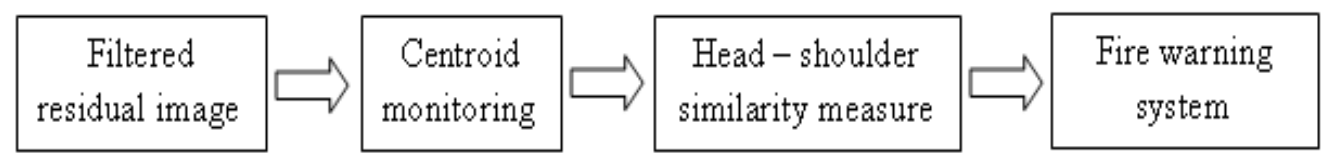

Fig. 6. Warning message generation

The system will extract information from the blobs and use it to determine action need to be taken. In this approach, the centroid of each blob will be used to determine whether the object is moving or relatively stay still at the same position. The system will monitor the position of centroid which will be compared frame by frame. When the distance is exceed the threshold value, the system will categorized the blob as moving blob and static blob when the distance is smaller than the threshold value. Fig. 6 above is used to illustrate the flow of warning or alert generation mechanism used in our approach.

As the system determines whether the blob is moving or static, the displacement of each centroid also monitored to determine whether the blob is moving or static. System will run head-shoulder similarity check when there is a blob that keeps static for certain period of time. The similarity check is done in attempt to detect a head and shoulder that belong to static object. Head shoulder detection is done through chain code [15-16]. This chain code is describe the boundary of the static blobs, which will be compared with predefined image that work as reference. These two chain code series is compared using Chain Code Histogram [17] and Chain Code Distance Vector.

\section{B. Database Module}

Database can be defined as a shared, integrated computer structure that stores data [18]. Database is managed through database management system (DBMS) such as MySQL, Oracle and IBM DB2 [19]. DBMS is used to make the data management more effective and efficient, it also used in order to share the data among multiple user. Therefore, database system need to be designed carefully in order to deliver good performance and efficient data management.

Database can be illustrated like a file cabinet. A file cabinet may has several drawer, which each drawer will contain several record inside it. Those records are uniquely identified by certain value where inside it, the related information can be found. The table will contain many data that categories in several field. Those data is uniquely identified by certain field called primary key. This primary key can be in form of ID, serial number, name or any other information that can be used to identify each record.

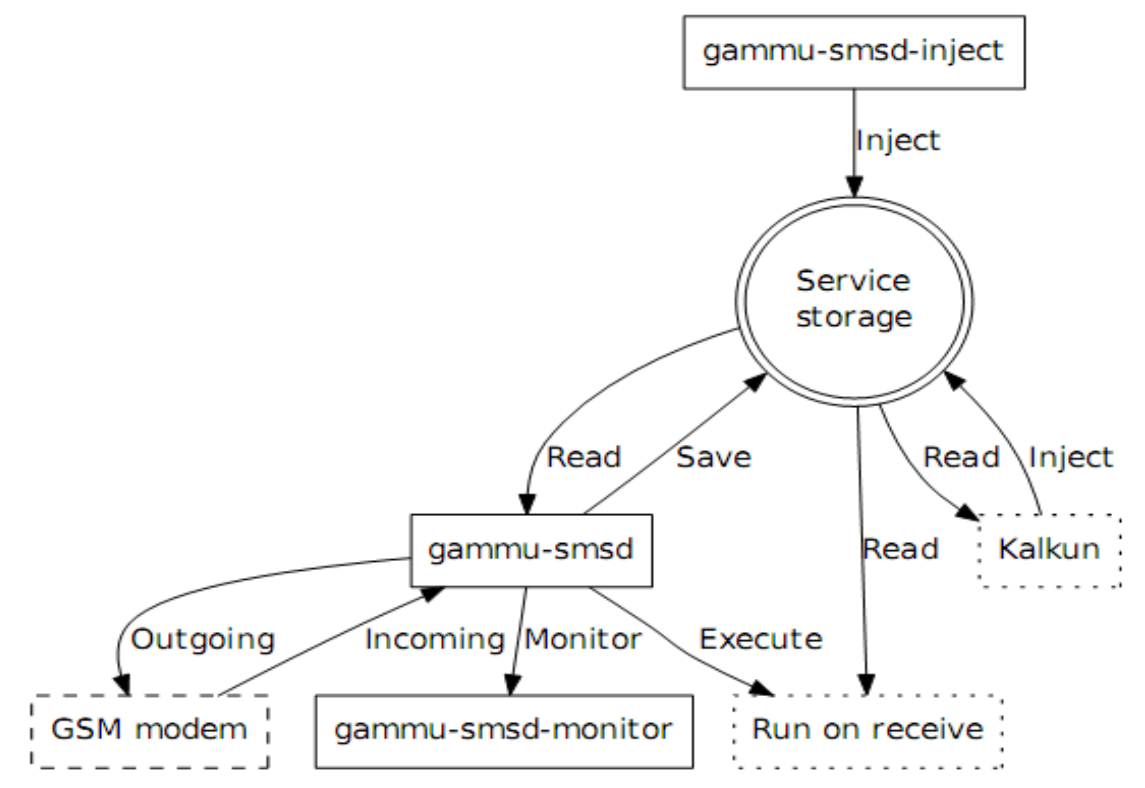

Fig. 7. Gammu working scheme 
Gammu is an application that provides abstract layer for cell phone access [20]. It is developed based on MyGnooki2 software and released under GNU GPL Version 2 which makes this program is free to share and change for all its users. Gaтmu provides library and command line utility for mobile phones to the user. This program will work with a database and a GSM modem to perform its tasks. GSM modem is a modem which accept SIM card and operates through a subscription to mobile operator like mobile phone. In order to handle incoming message, gammu will check GSM modem for any received messages and store those messages into database. This task is performed through gammu-smsdmonitor tool which monitor the status of SMS Daemon. For outgoing message, gammu will check if there is a queue message in the database. If there is any message need to be sent, gammu-smsd-inject tool is used to create a text messages which will be sent out through connected GSM modem. The working scheme of gammu can be seen in Fig. 7. From image processing module, any existing static head and shoulder will trigger the warning mechanism, to alert the owner of the vehicle that there is a suspicious activity around his vehicle. The warning mechanism consists of a database and GSM modem.

A record will be inserted into the database when static object is detected for certain period of time. The database set up during the simulation is mysql database that construct through wampserver. This database in automatically construct by gaтmu. Any new record in the database will trigger gammu to send a SMS based on the incoming record in the database. At the same time, the system will save the most recent frame and send it to the owner of vehicle to his e-mail. Through this step, owner of the vehicle will receive two notifications from the system. The first notification is through SMS and the other notification is through e-mail which also provides visual report of the current scene. Surveillance system also used a website to enable centralized monitoring system. Through this monitoring website, the latest scene is displayed together with the status of the vehicle whether it is safe or suspicious activity has been detected.

\section{RESULTS AND DISCUSSION}

At the first stage, system starts to receive stream of video. By assuming there is no object in the current frame, the first frame of the video will be used as the reference image which simulates the first image captured when the system becomes active. The entire incoming frame will be converted to grayscale image, it is done to make the process faster and more efficient. At this stage, ROI is also defined and applied on the image. ROI also can be used to filter out activities or changes that occur outside the expected region. It is becomes useful in this project since the activities near the vehicle may affect region that laid outside the expected region. Filtering out those changes make the system work with less computation load and work with better precision. Fig. 8 shows ROI implemented on the background image.

Beside noises reduction, the effect of different threshold value also can be seen in the main blob or silhouette of the person. In residual image with higher threshold value, more holes are observed on the blob. Less holes or more compact blob is seen on residual image with smaller thresh-old value. Morphological operation used here is dilation followed by erosion. Filtering process aim to remove any noises that still visible on the residual image. Also to remove blob that belong to object that not located beside the vehicle.

Morphological operation always used element structure on its process. In this task, the structuring element used is disk structure. Disk structure is chosen since disk shape can be said does not have strict shape, therefore can cover more flexible condition. Other than structure element itself, morphological operation also determined by the size of structure element. After performing morphological operations, the filtering process is applied to remove any blobs that can be categorized as noise or not potential threat to vehicle. Several criteria are used to filter out several blobs. Those criteria are blob size, blob height width ratio, and position of blobs in the ROI. Fig. 9 shows detect-ed object boundaries before and after size filtering.

A scene where there is a person walking beside the monitored a vehicle is illustrated in sequence of frames as shown in Fig. 10 with moving object is indicate by bounding box around it and the object's centroid. Another simulated situation is when a person walk beside the monitored vehicle, and then the person stop and look inside the vehicle as shown in sequences of frames in Fig. 11. A head-shoulder detection is activated when the system detect a static object for a pre-defined

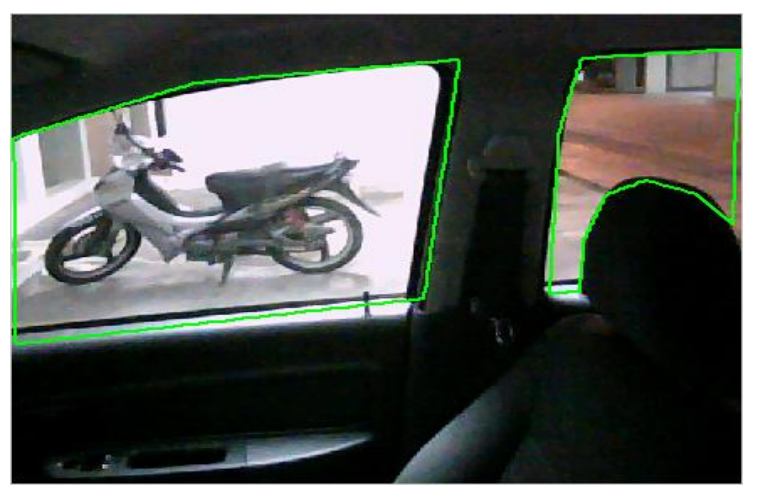

Fig. 8. Region of interest 
moment. If a head-shoulder is detected, the system will record the scene and generate a warning to notify the owner of vehicle. The notification is send through SMS

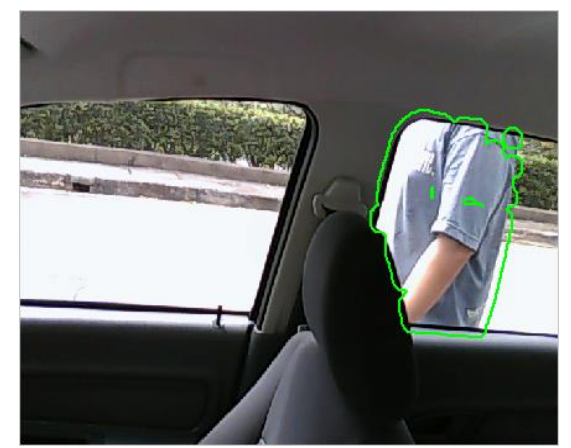

(a) and the owner will able to view the recorded image through email. Recorded image also can be used for investigation purpose by authorize person.

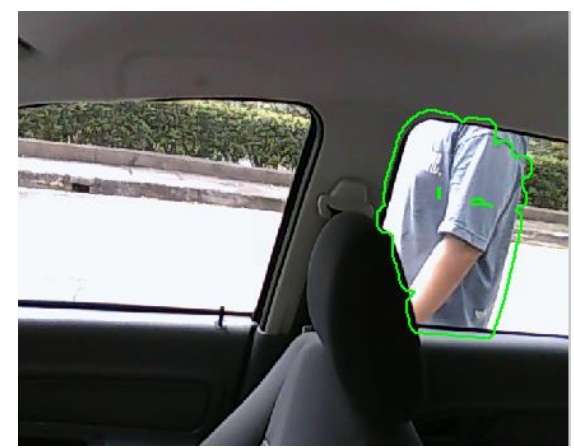

(b)

Fig. 9 Blob boundary: (a) before size filtering, (b) after size filtering

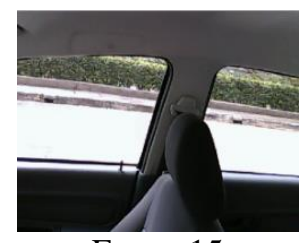

Frame 15

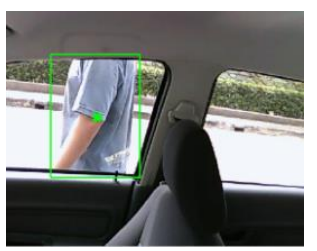

Frame 105

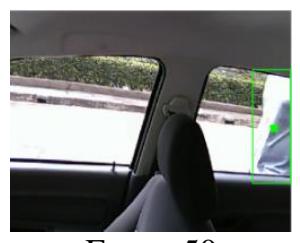

Frame 50

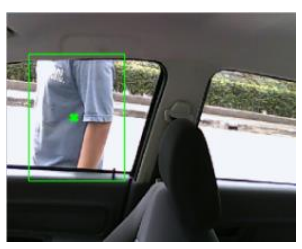

Frame 135

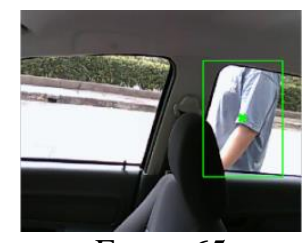

Frame 65

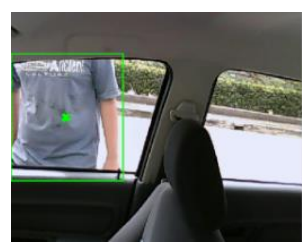

Frame 155

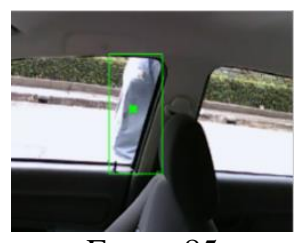

Frame 85

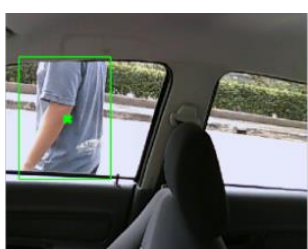

Frame 260

Fig. 10 Moving human object passes by the vehicle

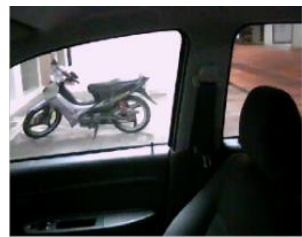

Frame 10

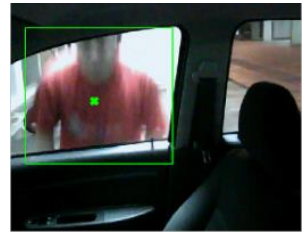

Frame 49

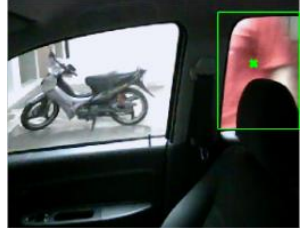

Frame 29

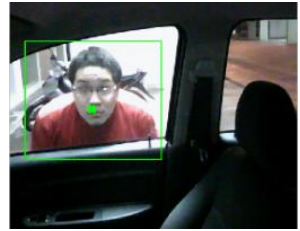

Frame 56

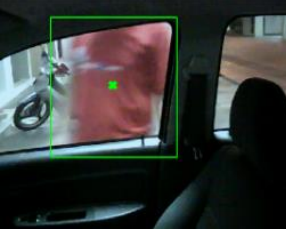

Frame 37

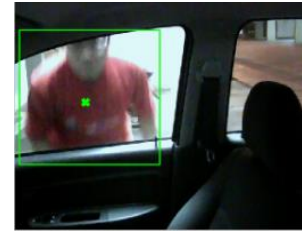

Frame 80

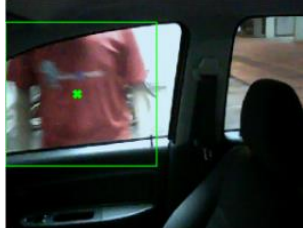

Frame 45

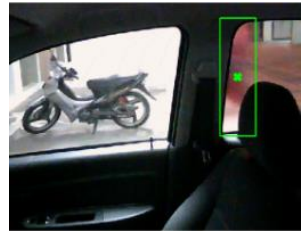

Frame 88

Fig. 11. Moving human object passes by, stop and look inside the vehicle 


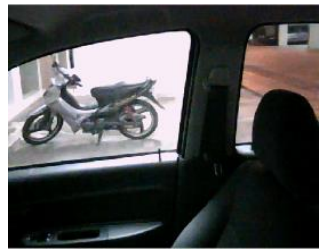

Frame 5

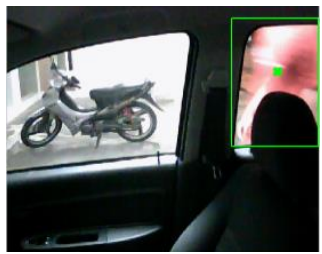

Frame 55

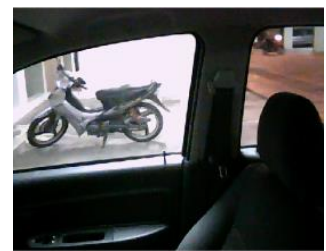

Frame 15

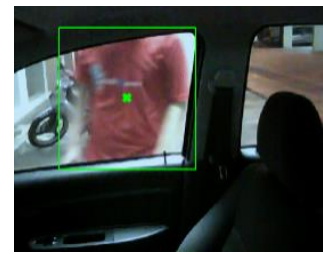

Frame 65

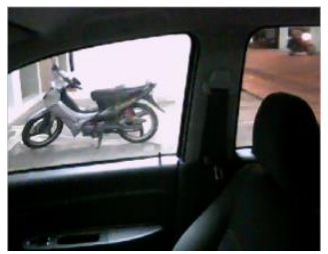

Frame 25

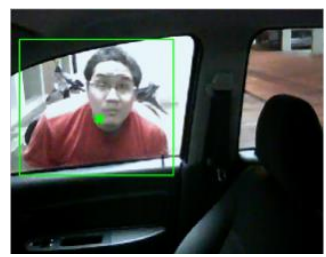

Frame 85

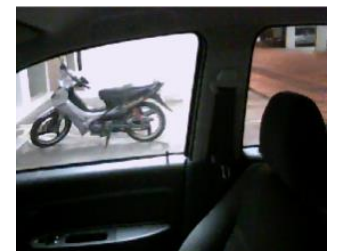

Frame 35

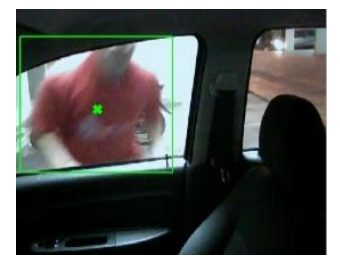

Frame 95

Fig. 12. Moving human object passes by, along with other moving vehicle and motorcycle

In Fig. 12, sequence of frames is shown to illustrate the moving object detection where there is two meaningless moving objects and one suspicious activity. In this video, there is a motorcycle that pass by and a car that moving out form its car park (at the upper-right position). These kind of movement not happened near the vehicle, so the system will ignore these movement as they are less possible to do any harm to the monitored vehicle. The other movement recorded is movement of a person that walk beside the car and look inside the car. This action can be categorized as suspicious activity that possibly do any harm to the vehicle.

First aspect that monitored by the system on warning mechanism is the centroid of the moving human object. Centroid is used since it marks the central point of the blob, therefore slight changes in blob shape or position will not cause many changes in centroid position.

Centroid monitoring may enable the system to keep track the object around the vehicle, but it is not enough to conclude that certain object can be categorized as suspicious activity. Therefore the system employs another aspect which is head shoulder detection. The head and shoulder detection is done using chain code. Chain code will provide the series of code to the system which describe the boundary line of a blob. The problem may arise when boundary shape is has similar shape as reference shape or it has chain code series that has similar code with reference code. Therefore, predefined similarity value is set to $40 \%$ similarity index. This value is set to still able to capture the blob that actually has head - shoulder on it, but has not similar chain series. When series of chain code satisfy the similarity level, the system will categorize the blob of having head and shoulder on it. At the same time, warning notification will be sent to the owner.

Warning notification is sent through SMS and the captured image is sent through e-mail service. The option of SMS is chosen since it will reach the owner almost everywhere and will take small amount of time to reach, where the e-mail provides the owner with ability to view the latest scene that trigger the warning notification. The status of vehicle and captured image also able to be monitored through centralized website. Fig. 13(a) is an example of warning notification which was sent through e-mail, while Fig. 13(b) shows an example of SMS notification send to the owner.

Besides sending warning to the owner of vehicle, system also employs a centralized website to monitor the situation of the vehicles. The website will show the latest scene from the vehicle and the status of vehicle, either it is safe or in danger. Through this website, centralized management will be able to view the recorded images if needed. This website can be used to send emergency message to those who have rights to take action if criminal activity is done such as police. The images stored also can be useful for investigation process or used as proof in court. Fig. 14 is showing a screenshot of website interface that used to display the information of vehicle owner, latest image and the time when image was taken. 


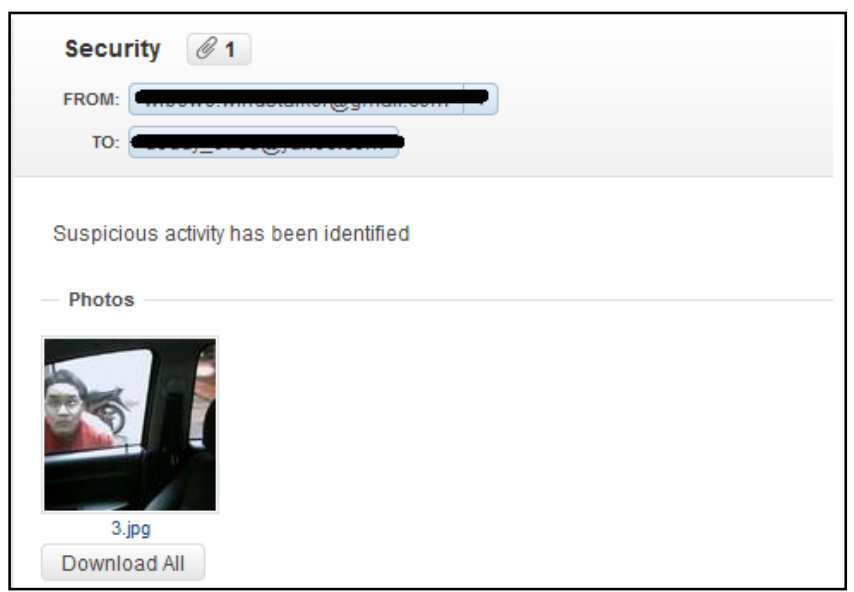

(a)

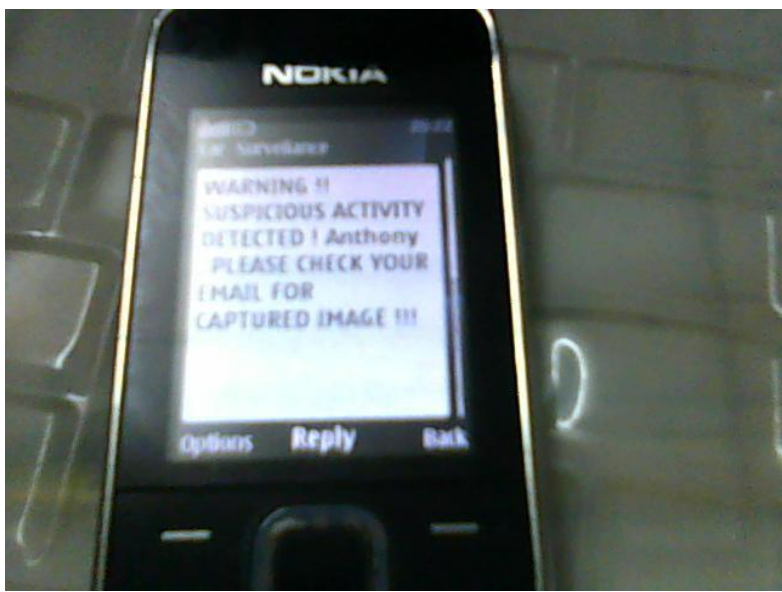

(b)

Fig. 13 Alert generation: (a) e-mail notification, (b) SMS

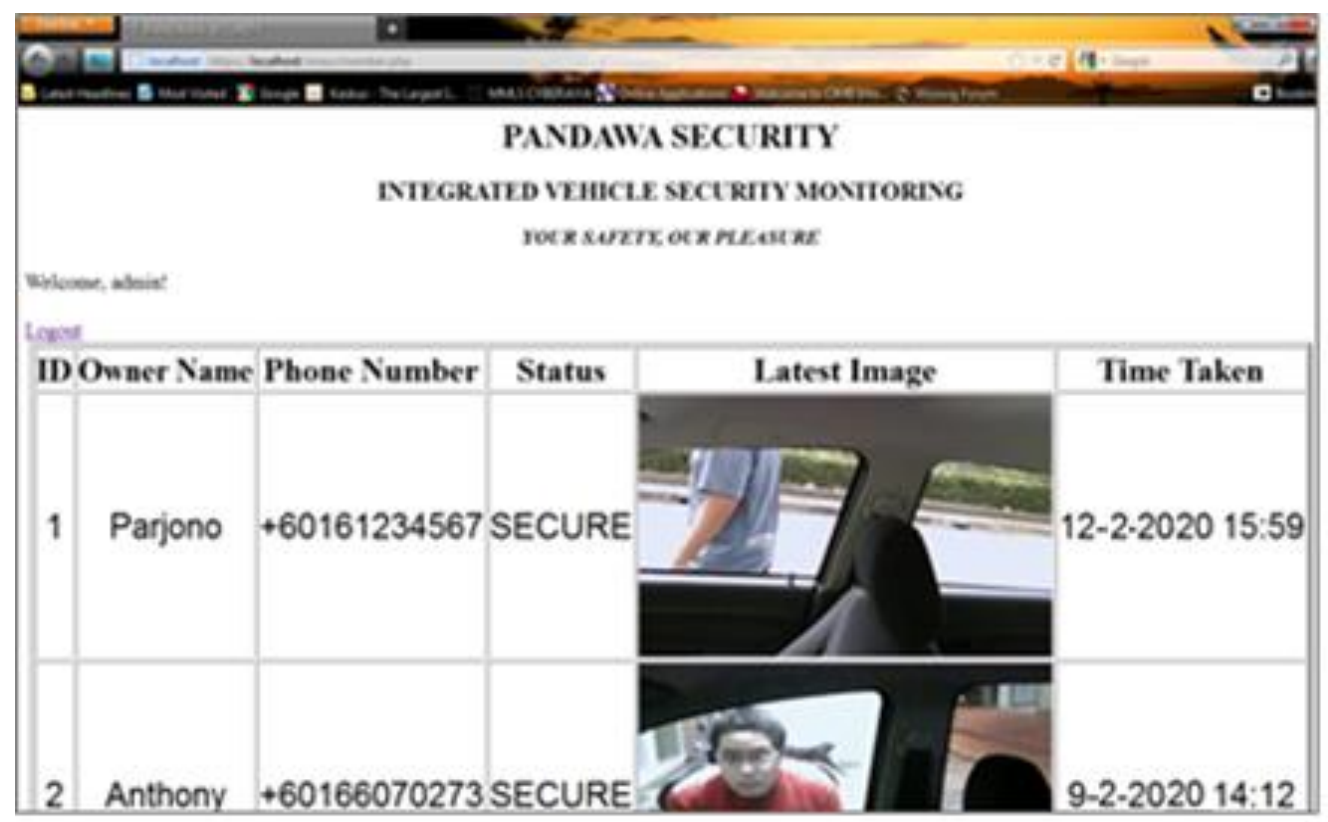

Fig. 14 Website for centralized monitoring system

\section{CONCLUSION}

One of the main feature of the proposed algorithm that make it different from other existing systems is that it can give some notification even before any intruders get into or break out the vehicle. Image of the suspected person is also can be known in an instant manner. Another important feature is on the integrated and organized information system in the form of website that enable the authority to keep track and monitor any suspected activities in a particular area. Some recommendations for future works can be used to improve the system in the future development. In motion detection stage, the system can be improved to be more robust especially in facing change of lights and shadow.
Improvement also could be done in blob analysis stage. Blob analysis can be improved by employing other filtering criteria and alternative operation to make blobs processing better. In warning mechanism, improvement could be done in suspicious activity detection. This detection scheme is important to avoid any criminal activity and to be able to capture the image from scene that can be used as valid proof in police investigation. Detection scheme may employ face detection or combine with face recognition which will enable the system to know whether the person is the owner's family or stranger. The surveillance system also may use GPS to enhance the surveillance system. This GPS may become useful when something happened with the vehicle that 
requires the authorized people to track the location of the vehicle. Other possible improvement is integration between vehicle surveillance systems. This integration will create such a surveillance network among the vehicle which may provide information when the respective vehicle cannot provide.

\section{ACKNOWLEDGMENT}

The authors would like to gratitude Malaysian Institute of Information Technology, Universiti Kuala Lumpur, Malaysia and Monster Technologies Sdn Bhd, Kuala Lumpur, Malaysia for providing any supporting facilities and conducive environment that makes this research possible and wonderful along the way.

\section{REFERENCES}

[1] M. Carvalho, "No. 6 on the world's top car theft list," The Star, Aug. 25, 2016. https://www.thestar.com.my/news/nation/2016/08/25/no -6-on-the-worlds-top-car-theft-list-about-60-vehiclesstolen-everyday-from-all-over-malaysia-says/. (accessed Feb. 21, 2021).

[2] J. P. Jeong and T. T. Oh, "Survey on protocols and applications for vehicular sensor networks," International Journal of Distributed Sensor Networks, vol. 12, no. 8, Aug. 2016.

[3] Y. Hori, Y. Sasaki, I. Miyamatsu, and S. Yakura, "Development of Intrusion Detection Sensor for Vehicle Anti-theft Systems," Fujitsu Ten Tech. J., no. 23, pp. 2631, 2000.

[4] K. P. Tanna, P. Kumar, and S. Narayanan, "Instant Theft Alert and Tracking System in Car," International Journal of Computer Applications, vol. 1, no. 21, pp. 975-8887, 2010.

[5] H. S. Kaashif, H. J. J. Antony, S. R. Raj, and D. Nithya, "Automobile Intrusion Avoidance Using Face Detection and Finger Print," International Journal of Computer Applications, no. 2, pp. 2278-8948, 2013.

[6] Z. Tufail, K. Khurshid, A. Salman, I. F. Nizami, K. Khurshid, and B. Jeon, "Improved Dark Channel Prior for Image Defogging Using RGB and $\mathrm{YCbCr}$ Color Space," IEEE Access, vol. 6, pp. 32576-32587, Jun. 2018.

[7] R. C. Gonzalez and R. E. Woods, Digital Image Processing, 4th ed. Pearson, 2018.
[8] I. Zeger, S. Grgic, J. Vukovic, and G. Sisul, "Grayscale Image Colorization Methods: Overview and Evaluation," IEEE Access, vol. 9, pp. 113326-113346, 2021.

[9] K. J. Chen and S. Y. Fang, "Printability Enhancement with Color Balancing for Multiple Patterning Lithography," IEEE Transactions on Emerging Topics in Computing, vol. 7, no. 2, pp. 244-252, 2019.

[10] F. Gasparini and R. Schettini, "Color balancing of digital photos using simple image statistics," Pattern Recognition, vol. 37, no. 6, pp. 1201-1217, 2004.

[11] P. M. Jodoin, J. Konrad, and V. Saligrama, "Modeling background activity for behavior subtraction," presented at the 2008 2nd ACM/IEEE International Conference on Distributed Smart Cameras, ICDSC 2008, 2008.

[12] J. Su, "Illuminant Estimation: Gray World," 2015. https://web.stanford.edu/ sujason/ColorBalancing/gray world.html (accessed Feb. 21, 2021).

[13] G. Boato, D. T. Dang-Nguyen, and F. G. B. D. Natale, "Morphological Filter Detector for Image Forensics Applications," IEEE Access, vol. 8, pp. 13549-13560, 2020.

[14] C. Solomon and T. Breckon, Fundamentals of Digital Image Processing: A Practical Approach with Examples in MATLAB. Wiley-Blackwell, 2012.

[15] H. Haron, S. M. Shamsuddin, and D. Mohamed, "Chain Code Algorithm in Deriving T-Junction and Region of a Freehand Sketch," Jurnal Teknologi, Jan. 2012.

[16] J. Sun and X. Wu, "Chain code distribution-based image retrieval," in 2006 International Conference on Intelligent Information Hiding and Multimedia Signal Processing, IIH-MSP 2006, 2006, pp. 139-142.

[17] Y. Xie, P. K. Hopke, G. Casuccio, and B. Henderson, "Use of chain code histogram method to quantify airborne particle shapes," Aerosol Science and Technology, vol. 21, no. 3, pp. 210-218, Jan. 1994.

[18] C. Coronel and S. Morris, Database Systems Design, Implementation, \& Management, 13th ed. Cengage, 2019.

[19] H. J. Bhatti and B. B. Rad, "Databases in Cloud Computing: A Literature Review," International Journal of Information Technology and Computer Science, vol. 9, no. 4, pp. 9-17, Apr. 2017.

[20] M. Čihař, "The Gammu Manual," 2015. https://docs.gammu.org/ (accessed Feb. 22, 2021). 
JUITA: Jurnal Informatika e-ISSN: 2579-8901; Vol. 9, No. 2, November 2021 